\title{
Highlights of ES2DE and IWOBI 2017: extended versions of selected best papers
}

\author{
Lucas Pereira ${ }^{1}$ - Antonio G. Ravelo-García ${ }^{2}$
}

Published online: 26 September 2018

๑) Springer-Verlag GmbH Austria, part of Springer Nature 2018

Computation has been developed in systems and devices with important evolutions in social and technological aspects that have allowed to solve different problems. In this issue, different computational solutions for challenges related to energy, wearable devices, image processing and computer vision are presented.

The special issue is composed by six papers, including this editorial, and is the result of a selection of the best papers from the following conferences that took place in Funchal, Portugal, July 2017: the IEEE Energy and Sustainability in Small Developing Economies (ES2DE), and the IEEE International Conference and Workshop on Bioinspired Intelligence (IWOBI).

In the article entitled "Optimal Electrical Power Generation with Underwater Kite Systems" [1], the authors investigate the use of Underwater Kite Power Systems (UKPS) as a mean to generate electricity from ocean or river tidal streams. To this end, the authors formulate an optimal control problem to find the trajectories and controls of the kite that maximize the total energy produced in a given time interval. In order to solve the optimization problem, a numerical solution was developed based on direct methods (in which the problem is discretized and transcribed into a static nonlinear programming problem). Simulations have shown that the problem can be successfully solved, and that it is possible to produce electric energy from such devices. To state more concretely, the simulations report an average power generation of $12.5 \mathrm{~kW}$ (with instant peaks up to $20 \mathrm{~kW}$ ) for a $2 \mathrm{~m}^{2}$ kite in a water stream of $5 \mathrm{~ms}^{-1}$.

In the article entitled "Finding Correlations of Features Affecting Energy Consumption and Performance of Web Servers Using the HADAS Eco-Assistant" [2], the authors present an extension of the HADAS platform. HADAS is a repository and eco-assistant targeted and assisting software researchers and developers in the analysis of the energy consumption of software solutions. The original system was

$凶 \quad$ Lucas Pereira

lucas.pereira@m-iti.org

1 Madeira Interactive Technologies Institute, Laboratory of Robotics and Engineering Systems, 9020-105 Funchal, Portugal

2 Institute for Technological Development and Innovation in Communications, Universidad de Las Palmas de Gran Canaria, 35017, Las Palmas de Gran Canaria, Spain 
extended by adding Pearson's Chi squared differentials and Bootstrapping statistics, to automatically check the significance of correlations of the energy consumption, and/or the execution time, with other variables that can influence the selection of a particular eco-efficient configuration. To evaluate the proposed extension the authors performed a sustainability analysis of five web-server configurations using time and energy measurements previously dumped in HADAS. Ultimately, this evaluation highlighted the potential of HADAS to provide clear pictures of the different trade-offs between energy consumption and execution time of different server configurations, which are key when conducting software sustainability analysis.

In the article entitled "Assessment of the spectral quality of fused images using the CIEDE2000 distance" [3], the authors investigate the use of the CIEDE2000 colordifference formula as a metric to evaluate the spectral quality of pan-sharpened images. To achieve this, the authors compared it with two classic spectral quality indices: Spectral Angle Mapper (SAM) and Mean Square Error (RMSE), and a qualitative measure of quality using a Mean Opinion test Score (MOS). The metrics were calculated using a dataset, composed of four satellite images, each of which was pan-sharpened using 9 algorithms. The resulting 36 pan-sharpened images were used to calculate the quantitative metrics. As for the MOS, a test was carried out by collecting the opinions of 10 expert users that were asked to rate the spectral quality of each pan-sharpened image on a scale of 5-1 points. Since CIEDE2000 was adapted to the perception of the human visual system, the basis assumption was that the results of quantitative spectral quality assessment would match with those a human observer would note. Ultimately, the obtained results led to the conclusion that the evaluations carried out with RMSE and SAM did not agree with those obtained by the MOS test. In contrasts, there was a very high correlation between CIEDE2000 and the results of the MOS tests, thus confirming the initial assumption of the authors.

In the article entitled "Computer Vision Based Technique for Identification and Quantification of Powdery Mildew Disease in Cherry Leaves" [4], the authors proposed a method to decide (healthy or disease) in cherry leaves. The authors proposed a method using adaptive intensity-based thresholding for automatic segmentation of powdery mildew disease which makes the method invariant to image quality and noise. The method is tested on the comprehensive dataset of leaf images of cherry crops, which achieved an accuracy of $99 \%$. The authors concluded that the method for segmentation of powdery mildew disease affected area from leaf image of cherry crops is convincing and computationally cheap.

In the article entitled "A portable wireless device based on oximetry for sleep apnea detection" [5], the authors designed and evaluated a portable and automated sleep apnea detector based on a $\mathrm{SpO} 2$ sensor. A field-programmable gate array receives the signal and checks for errors implementing the communication protocols of two wireless transmitters. Two solutions were implemented: one based on a smartphone and another based on a personal computer. Both implementations have a graphical user interface and the algorithms were tested using a database consisting of 70 patients with the $\mathrm{SpO} 2$ recording. The algorithm performance achieved an average accuracy, sensitivity and specificity of $87.5,79.5$ and $90.8 \%$ respectively. 


\section{References}

1. Paiva LT, Fontes FACC (2018) Computing. https://doi.org/10.1007/s00607-018-0643-4

2. Munoz DJ, Pinto M, Fuentes L (2018) Computing. https://doi.org/10.1007/s00607-018-0632-7

3. Rodríguez-Esparragón D, Marcello J, Gonzalo-Martín C et al (2018) Computing. https://doi.org/10.10 07/s00607-018-0640-7

4. Sengar N, Dutta MK, Travieso CM (2018) Computing. https://doi.org/10.1007/s00607-018-0638-1

5. Mendonça F, Mostafa SS, Morgado-Dias F et al (2018) Computing. https://doi.org/10.1007/s00607-01 8-0624-7 